\begin{tabular}{|c|l|}
\hline Title & Flow transitions in the surface switching of rotating fluid \\
\hline Author(s) & Tasaka, Y.; Iima, M. \\
\hline Citation & Journal of Fluid Mechanics, 636, 475-484 \\
\hline https:/doi.org/40.1017/S0022112009991005 \\
\hline Issue Date & 2009-10 \\
\hline Doc URL & http://hdl.handle.net/2115/39840 \\
\hline Rights & ○ Cambridge University Press 2009 \\
\hline Type & article \\
\hline File Information & JFM636_p475-484.pdf \\
\hline
\end{tabular}

Instructions for use 


\title{
Flow transitions in the surface switching of rotating fluid
}

\author{
Y. TASAKA ${ }^{1} \uparrow$ AND M. II MA ${ }^{2}$ \\ ${ }^{1}$ Graduate School of Engineering, Hokkaido University, N13W8, Sapporo 060-8628, Japan \\ ${ }^{2}$ Research Institute for Electronic Science, Hokkaido University, N20W10, Sapporo 001-0020, Japan
}

(Received 26 March 2009; revised 25 June 2009; accepted 26 June 2009)

We study 'surface switching' quantitatively in flows driven by the constant rotation of the endwall of an open cylindrical vessel reported by Suzuki, Iima \& Hayase (Phys. Fluids, vol. 18, 2006, p. 101701): the deformed free surface switches between axisymmetric and non-axisymmetric shapes accompanied by irregular vertical oscillation. Detailed simultaneous measurements showed that the magnitude of the velocity fluctuations (turbulent intensity) temporally varies greatly and are strongly correlated with the surface height, suggesting that dynamic switching between laminar and turbulent states is accompanied by vessel-scale surface shape changes. The study also identified clear hysteresis in the turbulent intensity arising from changes in the Reynolds number; the bifurcation diagram consists of two overlapping branches representing a high-intensity (turbulent) state and a low-intensity (laminar) state. Based on the results, a switching mechanism is suggested.

\section{Introduction}

The free surface shape of fluids is determined by flow characteristics. Even in the simplest situation, like in flows driven by a rotating endwall of a stationary cylinder that is open at the top (cf. figure 3), the surface shape is strongly influenced by parameters such as the cylinder size, rotating speed, fluid volume, surface tension and other parameters. This kind of system has been studied extensively (Lopez et al. 2004; Jansson et al. 2006; Suzuki, Iima \& Hayase 2006; Shatrov, Gerbeth \& Hermann 2008), as has the surface shape arising from granular flow (Corwin 2008).

At small rotation rates, the flow is symmetrical like the cylindrical container it occurs in, but the symmetrical shape breaks down at Reynolds numbers $(R e)$ around 2000 (here $R e=2 \pi \Omega R^{2} / \nu$, where $\Omega$ is the rotating speed of the disk, $R$ the radius of the disk and $v$ the kinematic viscosity of the fluid) (Lopez et al. 2004). In this case, the deformation of the free surface is quite small and free surface deformation effects can be ignored. At large rotating speeds, the deformation becomes comparable to the scale of the vessel. In such cases, the horizontal cross-sections of the deformed surface may be polygonal in shape (Jansson et al. 2006), with polygonal shapes observed when smaller disks are used (Vatistas 1990; Vatistas, Abderrahmane \& Siddiqui 2008), reporting temporally periodic oscillations of the surface shape between differently deformed surface shapes with polygonal cross-sections.

Suzuki et al. (2006) used a close-fitting disk, and found that at $\operatorname{Re} \sim 1.0 \times 10^{5}$ the deformation of the surface changed from one shape to another non-periodically.

$\dagger$ Email address for correspondence: tasaka@eng.hokudai.ac.jp 
They termed this phenomenon 'surface switching'. An irregular switching of the rotational symmetry occurs, together with vertical oscillation of the surface. During the switching process, an $n=2$ ( $n$ is the number of the apex) symmetry state was observed at relatively higher positions of the surface, and an $n=0$ symmetry state was observed at relatively lower positions of the free surface. Suzuki et al. (2006) suggested that there was an increase in the static pressure at the centre of the cylinder due to strong mixing of the flow, and that the pressure decreased when the flow reverted to laminar flow. Suzuki et al. (2006) also reported a finite jump in the mean surface height at the critical surface-switching Reynolds number, which suggests the occurrence of a flow transition. Visualization of the instantaneous flow field established that the flow in the switching process is turbulent at the $n=2$, and laminar at the $n=0$ state (Tasaka, Ito \& Iima 2008b).

Studies of the boundary layer on rotating disks here indicated that a transition of the boundary layer occurs at $R e \sim 10^{5}$ when the distance from the centre is considered the characteristic length (von Kármán 1921; Gregory, Stuart \& Walker 1955; Reed \& Saric 1989). This critical Reynolds number is of the same order as that for the onset of surface switching. In the transition to turbulence, a turbulent spot or turbulence on the spiral vortices first appears at the edge of the disk, and subsequently spreading to become global turbulence, as has been observed in the flow between rotating and stationary disks (Cross \& Le Gal 2002; Le Gal et al. 2007). In the set-up used in the paper here, the surface shape changes greatly during the switching process, and such a change would shift the critical Reynolds number. These facts lead us to expect that the flow transition of the boundary layer may play the role of a trigger for the initiation of the turbulent flow, and that the coupling between the flow transition and change in surface shape is a key factor in the mechanism of surface switching. We do not however have quantitative evidence to suggest a clear causative connection between the surface deformation and the global flow transition. The flow transition discussed here expresses the transition between a laminar and a wholly disturbed flow (termed as 'turbulent' below) in the dynamic process of switching.

This paper aims to clarify the correlation between the dynamic process of the surface switching and the flow transition. For this purpose, an experimental set-up, an improvement on that in Suzuki et al. (2006) was used to enable more detailed measurements as explained below. The results show that the turbulent intensity displays hysteresis in relation to the change in the Reynolds number, which has not so far been reported elsewhere. The transitions are described by a bifurcation diagram consisting of two partially overlapping branches corresponding to small and large turbulent intensities, and the region of the surface switching is adjacent to the overlapping region. Also, the surface height and the instantaneous velocity profiles were measured simultaneously, and it was found that the magnitude of the velocity fluctuation greatly varies in relation to the surface height changes. During the surface switching, we observed several quasi-steady states, including new so far undescribed states, which are similar to the steady-flow states observed in the neighbourhood of the Reynolds numbers where surface switching occurs. Finally, a switching mechanism is suggested based on the results.

\section{The surface switching}

Surface switching phenomena and related flow patterns are summarized in the following. Figure 1 shows the shape of a free surface and the flows visualized by Kalliroscope flakes (e.g. Tasaka et al. 2008b), a suspension of micro platelets that 


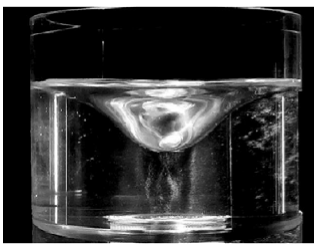

(a)

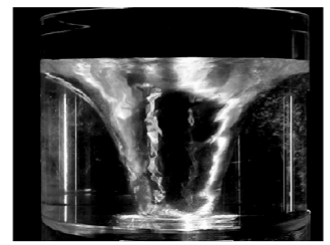

(b)

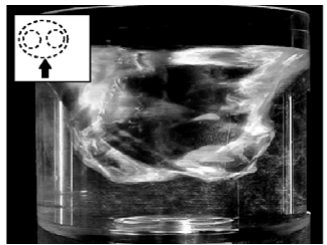

$(c)-1$

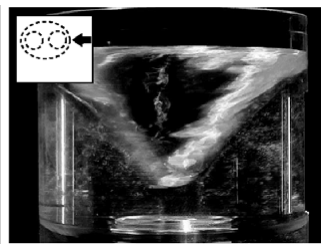

(c) -2

FiguRE 1. Photos of the visualized flow pattern at each state; $(a) R e=0.55 \times 10^{5}(S y)$, (b) $R e=1.11 \times 10^{5}\left(S y^{\prime}\right),(c) R e=1.56 \times 10^{5}(S w)$ ((c)-1 front view and $(c)-2$ side view). Suspended micro platelets are used for the visualization of shear flows. The flow pattern is laminar in $(a)$ and $(b)$, and turbulent in $(c)$.

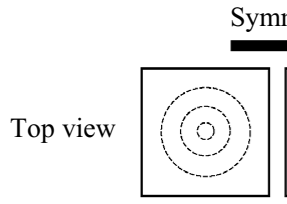

(a)

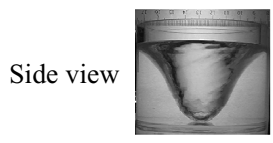

(b)

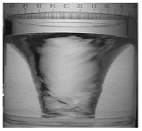

$(c)$

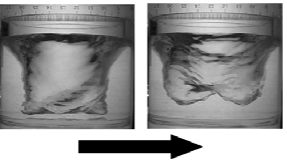

Bottom elevation

\section{Symmetry recovery}

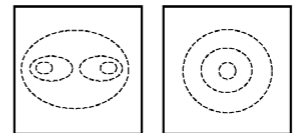

(e)

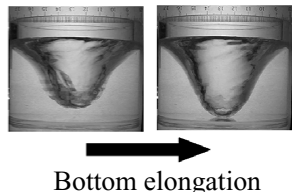

Figure 2. Typical sequence of the switching process. Top view: schematic representations of the horizontal cross-sections; side view: visualized free surface shapes. The free surface repeats the deformation sequence from $(a)$ to $(e)$.

make shear flows visible by the fine contrast of the brightness in images. A typical flow pattern where the rotating speed is relatively small $\left(\operatorname{Re}<\operatorname{Re}_{a}\left[=0.98 \times 10^{5}\right]\right)$ (Suzuki et al. 2006; Tasaka et al. 2008b) is similar to rigid body rotation near the rotating axis $(r<0.5 R ; r$ is the radial component) and here the free surface shape is close to a rotating paraboloid $(S y)$, as suggested by figure 1( $a)$. A typical flow pattern for higher rotating speeds $\left(R e_{a} \leqslant R e<R e_{c}\left[=1.40 \times 10^{5}\right] ; S y^{\prime}\right)$ is shown in figure $1(b)$ (Suzuki et al. 2006; Tasaka et al. 2008b). The rigid body motion near the centre has disappeared due to the attachment of the free surface to the bottom, but the flow state is still laminar in most regions of the bulk fluid. In a subset of an $S y^{\prime}$ region $\left(R e_{b}\left[=1.29 \times 10^{5}\right] \leqslant R e<R e_{c}\right)$, the axisymmetric surface shape intermittently becomes unstable and an $n=2$ surface deformation takes place (cf. figure 4)(Tasaka, Iima \& Ito 2008a).

Surface switching $(S w)$ is observed in the regime where $R e_{c} \leqslant R e<R e_{d}\left(=1.6 \times 10^{5}\right)$ (Suzuki et al. 2006; Tasaka et al. 2008b). A typical switching sequence proceeds as follows (figure 2): $(a)$ the bottom of the axisymmetric surface $(n=0)$ elongates and attaches to the rotating disk; $(b)$ the axisymmetry of the free surface breaks down and the transition to an $n=2$ shape occurs; $(c)$ the horizontal deformation of the free surface becomes larger and finally the surface is fully detached from the bottom; $(d)$ the bottom of the surface moves to a higher position and the surface rotates uniformly with an $n=2$ shape with two distinct humps (figure $1 c) ;(e)$ the free surface reverts to an axisymmetric shape and is elongated towards the bottom (resembling figure $2 a$ ). The visualized shear flows show that the flow state in figure $2(a)$ is similar to that in figure $1(b)$, it is laminar, and the flow state in figure $2(d)$ is turbulent, which is similar 


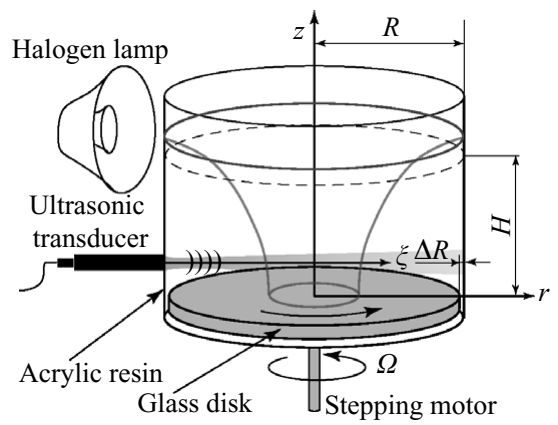

FIgURE 3. Experimental set-up for the UVP measurements and the flow visualization; the disk in the open cylindrical vessel is rotated by a stepping motor. An ultrasonic transducer is mounted at a height of $8 \mathrm{~mm}$ from the disk.

to that shown in figure 1(c) (Tasaka et al. 2008b). Therefore, both the laminar and turbulent states of flow occur during the switching process. The actual time evolution of the switching process consists of several quasi-steady states appearing irregularly. In particular, the elongation of the free surface does not always reach the rotating disk (cf. figure 5).

For higher rotating speeds $\left(R e \geqslant R e_{d}\right)$, the free surface with an $n=2$ shape stays at a higher position and does not extend to the bottom $(A s)$. In this condition a turbulent state is maintained while at the same time the free surface undergoes small oscillations with a period of about $10 \mathrm{~s}$, which is more than 100 times longer than the period of rotation. At higher speeds of rotation the surface is again attached to the bottom, and finally, the surface returns to the axisymmetric shape (Suzuki et al. 2006).

\section{Experimental set-up}

Figure 3 shows a coordinate system and a schematic outline of the experimental set-up. The open ended cylindrical vessel is made of acrylic resin; the inner radius is $R=42 \mathrm{~mm}$. A glass disk mounted at the bottom of the vessel is connected to a stepping motor through a shaft. It is possible to adjust the rotating speed of the disk in steps of 10 r.p.m. To reduce vibrations due to friction, there is a gap of $\Delta R=0.3 \mathrm{~mm}$ between the disk and the sidewall of the vessel. The rotating speed of the disk and the gap has been adjusted and improved, compared with the experimental set-up in Suzuki et al. (2006). The maximum tilt of the disk sitting on the shaft was 0.06 degrees, while such tilt was negligible in the set-up in Suzuki et al. (2006) because a cylinder disk rotor was used to drive the fluid. The vessel was filled with tap water; the liquid height at rest was $H=40 \mathrm{~mm}$; and the aspect ratio $\mathscr{A}=H / R=0.95$. The values of $R$ and $\mathscr{A}$ are close to the values of those in the experiments in Suzuki et al. (2006). Particles of porous resin (50-60 $\mu \mathrm{m}$ in diameter and $1020 \mathrm{~kg} \mathrm{~m}^{-3}$ in density), were mixed into the water as tracers for the velocity profile measurements. The rotating speed of the disk was varied from 300 to 850 r.p.m. In this range, the Reynolds number $R e$ varies from $0.55 \times 10^{5}$ to $1.57 \times 10^{5}$, corresponding to a range of Weber numbers, $W e=\rho(2 \pi \Omega)^{2} R^{3} / \sigma$ ( $\rho$ and $\sigma$ are density and surface tension of water, respectively) of $1.00 \times 10^{3} \leqslant W e \leqslant 8.06 \times 10^{3}$ and of Froude numbers, $F r=2 \pi \Omega R /(\mathrm{gH})^{1 / 2}(\mathrm{~g}$ is the gravity acceleration) and $2.11 \leqslant F r \leqslant 5.97$, respectively. The Reynolds number is used to describe the results examining the effect of the laminar-turbulent transition 


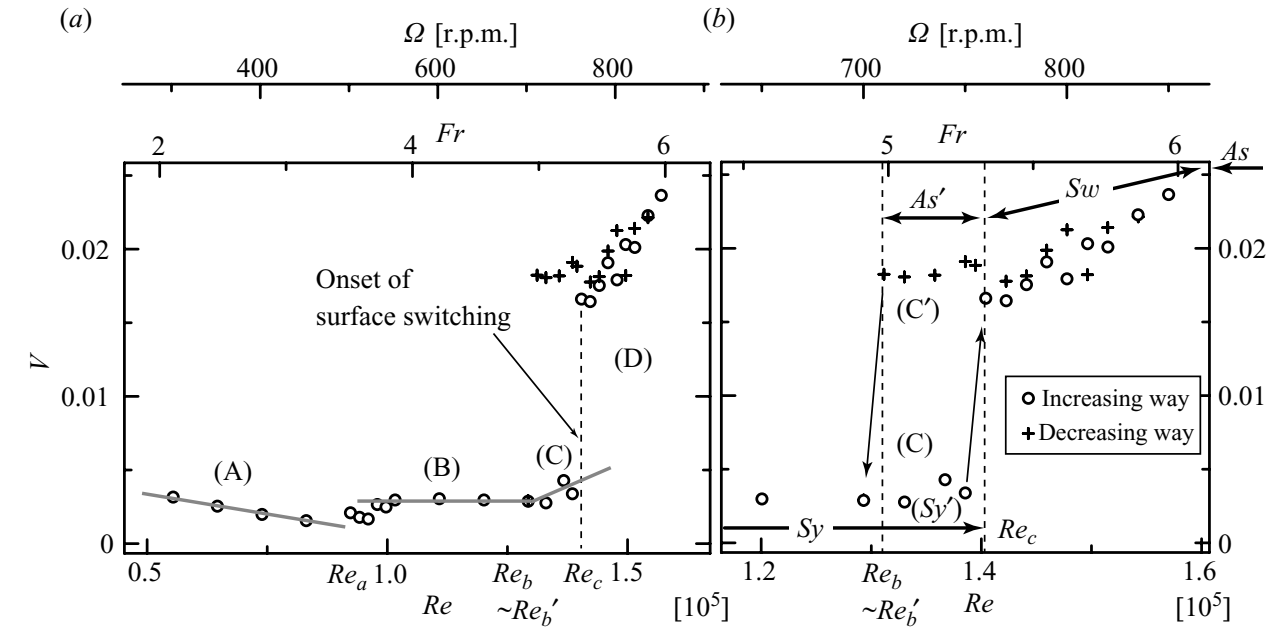

(b)

FIGURE 4. Turbulent intensity $V$ with increasing Reynolds numbers (open circles) and with decreasing Reynolds numbers (crosses). Rotating speed of the disk $(\Omega)$ and the Froude numbers $(F r)$ are also indicated. $(a)$ In region $(\mathrm{A})\left(\operatorname{Re}<R e_{a}\right)$, the free surface is not attached to the bottom. In region $(\mathrm{B})\left(R e_{a}<R e<R e_{b}\right)$, the free surface touches the bottom and there is no distinct instability observed. In region $(\mathrm{C})\left(R e_{b}<R e<R e_{c}\right)$ with increasing Reynolds numbers, the surface still touches the bottom, but intermittent surface instability is observed. In region $(\mathrm{D})\left(R e_{c}<R e\right)$, the surface has detached from the bottom, and surface switching is observed. (b) A magnification of a part of $(a)$. There is clear hysteresis in the region $R e_{b}^{\prime} \simeq R e_{b}<R e<R e_{c}$. The surface shapes in (C) are axisymmetric and touch the bottom. In $\left(\mathrm{C}^{\prime}\right)$ the surface shapes have $n=2$ symmetry and the average height is maintained at a higher position.

as suggested in Suzuki et al. (2006). Ultrasonic velocity profiling (UVP) was used to determine the instantaneous velocity profiles along the line of propagation of the ultrasonic waves. This is a useful tool to capture the spatiotemporal motion of fluids and to investigate statistical properties of flows (Takeda 1996; Mashiko et al. 2004; Tasaka et al. 2006). An ultrasonic transducer was mounted at the sidewall of the vessel perpendicular to the wall at a height of $8 \mathrm{~mm}$ from the disk $(z / H=0.2)$. The contact face of the vessel with the transducer was flattened to improve the transmission of the ultrasonic wave through the cylinder wall. Instantaneous profiles of the radial velocity component along the horizontal centreline of the cylinder $u(r, t)$ were measured through the sidewall. The basic frequency of the ultrasonic wave was set at $4 \mathrm{MHz}$; the spatial resolution of the velocity profile is $0.74 \mathrm{~mm}$. Further details of UVP are described in Takeda (1995). The number of velocity profiles is 4096 for $R e \geqslant R e_{c}$ and 1024 for $R e<R e_{c}$. The sampling period was varied from 13 to $34 \mathrm{~ms}$ depending on the flow. The spatial distribution of the intensity of the temporal velocity fluctuation, $u_{s p}(r)=\left[(1 / T) \int_{0}^{T} u^{\prime}(r, t)^{2} \mathrm{~d} t\right]^{1 / 2}$ (where $u^{\prime}(r, t)$ is the fluctuating component of $u(r, t)$ and $T$ is the measurement time), was calculated to describe the flow transition with respect to the Reynolds number. The time series of the spatial root mean square of $u^{\prime}(r, t), u_{r m s}(t)=\left[\left(1 /\left(\eta_{2}-\eta_{1}\right) R\right) \int_{\eta_{1} R}^{\eta_{2} R} u^{\prime}(r, t)^{2} \mathrm{~d} r\right]^{1 / 2}$ $\left(\eta_{1}=0.49, \eta_{2}=0.67\right)$ was also used to describe the dynamic switching quantitatively (cf. figure 5).

The free surface of the water was illuminated by back lighting (a halogen lamp with the light scattered by tracing paper) from the side of the cylinder, and its motion was captured by a digital video camera at 29.97 frame per second. 


\section{The flow transitions in the surface switching}

The process of the surface switching explained in $\S 2$ can be understood by expressing the turbulent transition in this system quantitatively. Figure 4 shows the turbulent intensity $V$ defined as the non-dimensionalized spatial average of $u_{s p}$, $V=1 /\left(2 \pi \Omega R\left(\eta_{2}-\eta_{1} R\right)\right) \int_{\eta_{1} R}^{\eta_{2} R} u_{s p}(r) \mathrm{d} r$, as a function of $R e$ (The values of $F r$ and $\Omega$ are also indicated in figure 4.); $F r$ is of the order of one in the present experimental condition and the centrifugal force is comparable to gravity. Hysteresis is clearly observed when increasing or decreasing $R e$ and either of two flow states may occur at $1.29 \times 10^{5}<R e<1.40 \times 10^{5}$ (700 r.p.m. $<\Omega<760$ r.p.m.).

The part of the lower turbulent intensity forms a branch (laminar branch). In this condition, the flow state is laminar (Tasaka et al. 2008b), while the velocity fluctuations due to the symmetry breaking and subsequent bifurcations (Lopez et al. 2004) contribute to $V$. The $V$ value decreases with increasing $R e$ within a small range, until the free surface attaches to the bottom of the vessel $\left(R e<R e_{a}\left[=0.98 \times 10^{5}\right.\right.$; $\Omega=530$ r.p.m.]) (figure $4 a$ ). In the range of $R e$, where the free surface has attached to the bottom, $V$ maintains a nearly constant value (figure $4 a$, region (B)). The interfacial instability reported by Tasaka et al. (2008a) occurs at $R e=\operatorname{Re}_{b}\left(=1.29 \times 10^{5}\right.$; 700 r.p.m.), where intermittent oscillation first appears. Due to this instability, $V$ increases slightly (figure $4 a$, region (C)). The intermittent oscillation enhances $V$, further causing large velocity fluctuations, and finally overcomes the suppression imposed by surface tension at $R e=R e_{c}$. The critical Reynolds number here depends on the material of the rotating disk: for glass (present study) it is $R e_{c}=1.40 \times 10^{5}$ (760 r.p.m.) and for acrylic resin it is $R e_{c}=1.56 \times 10^{5}$ (840 r.p.m.) (Tasaka et al. $2008 a$ ). Other critical Reynolds numbers are not sensitive to the materials employed. About the critical Reynolds numbers the free surface detaches from the bottom and the surface switching takes place (figure $4 a$, region (D)).

The part of the higher turbulent intensity forms a different branch (turbulent branch). The characteristics of the flow are represented by the power spectrum of $u^{\prime}(r, t)$ at radial positions (Tasaka et al. 2008a). There are three peaks at $f=f_{1}, f_{2}, f_{3}$, $\left(f_{1}>f_{2}>f_{3} ; f\right.$ the frequency) and combinations of these, although the peaks are with broad supports due to the turbulent flow. The peak corresponding to $f_{2}$ is for the $10 \mathrm{~s}$ scale oscillation and $f_{1}$ is for the rotation of the humps shown in figure $2(d)$.

In region $R e_{b}^{\prime}\left(=1.29 \times 10^{5}\right)<R e<R e_{c}$ (figure $4 b$, regions (C) and (C')), it is possible to achieve both the laminar and the turbulent states, depending on the initial conditions. The $\left(\mathrm{C}^{\prime}\right)$ state is achieved when the Reynolds number is decreasing from higher values; in this region, the turbulent intensity $V$ is approximately constant, and no surface switching is observed. The typical surface shape is similar to the $n=2$ symmetry state, but the humps are not very distinct. This state has not been reported previously and it is referred to as ' $A s^{\prime \prime}$ ' hereafter. As Re decreases, the $10 \mathrm{~s}$ scale oscillation amplitude of the free surface is reduced, and finally the flow becomes laminar with the surface attached to the bottom. The critical Reynolds number $R e_{b}^{\prime}$ here is close to $R e_{b}$ and the critical Reynolds number for the surface switching that was reported by Suzuki et al. (2006), who did not report a clear hysteresis in the surface height. A possible explanation of the simplified bifurcation structure in Suzuki et al. (2006) is a noise effect due to a different experimental set-up, which could blur the fine details of the bifurcation structure reported here. It is noteworthy that the surface shapes in the overlapping regions are different; axisymmetric and touching the bottom for the laminar branch, and, non-axisymmetric and not touching 


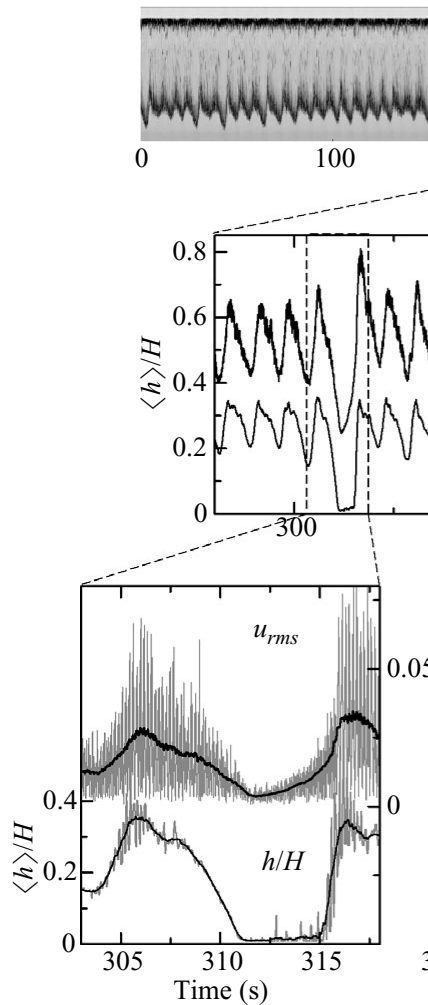

(a)

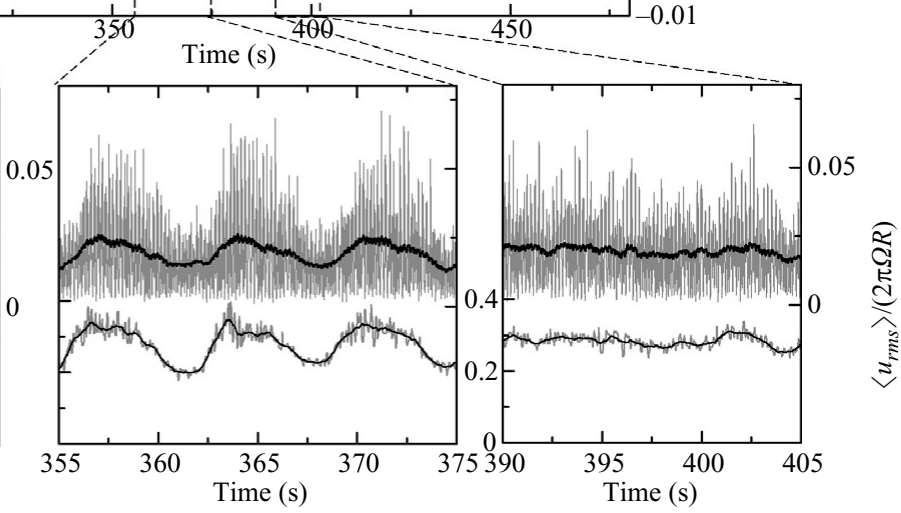

(b)

(c)

FIGURE 5. Variations in the surface height and corresponding velocity fluctuations in the switching process. Top: Temporally expanded image of the free surface extracted at the centreline of the cylinder. Middle: Time series of $0.53 \mathrm{~s}$ moving average surface height $(\langle h\rangle)$ and $0.52 \mathrm{~s}$ moving average of $u_{r m s}\left(\left\langle u_{r m s}\right\rangle\right)$. Bottom: Enlargements corresponding to $(a)$ laminar turbulent switching (LTS), $(b)$ a part of regular oscillation (RO) and (c) flat rotation (FR). The grey lines represent the measured fluctuations $\left(R e=1.46 \times 10^{5}\right)$.

to the bottom for the turbulent branch. The differences in the shapes result from the differences in the critical Reynolds numbers for the laminar-to-turbulent and the turbulent-to-laminar transitions.

\section{Three modes in the surface switching}

The following analyses the dynamic processes involved in surface switching based on the simultaneous measurements of surface height and velocity field.

Figure 5 shows the dynamic flow transitions in surface switching $\left(R e=1.46 \times 10^{5}\right.$; $\Omega=790$ r.p.m.). Figure 5 (top) shows the changes in the surface height $h(t)$ for a $500 \mathrm{~s}$ period. Figure 5 (middle) shows numerical data for $h(t)$ and the turbulent intensity of the corresponding velocity fluctuation, $u_{r m s}(t)$ for a part of the data in figure 5 (top). The curves in figure 5 (middle) are $0.53 \mathrm{~s}$ moving average surface height $(\langle h\rangle)$ and 0.52 s moving average of $u_{r m s}\left(\left\langle u_{r m s}\right\rangle\right)$, respectively. Moving averages are adopted to remove short-term fluctuations. In figure 5 (bottom), there are typical dynamic sequences; grey lines ( $h$ and $u_{r m s}$ ), and the moving averages $\left(\langle h\rangle\right.$ and $\left.\left\langle u_{r m s}\right\rangle\right)$ superimposed, representing different modes: laminar-turbulent switching (LTS) (figure 5a), regular oscillation (RO) (figure 5b) and flat rotation (FR) (figure 5c). 
The LTS is a typical phenomenon reported by Suzuki et al. (2006), a temporal switching of the surface between axisymmetric and non-axisymmetric shapes, with large vertical motion. The typical sequence and the flow structure have been summarized in $\S 2$ above. Typical LTS sequences are observed at $310 \mathrm{~s}<t<320$ $\mathrm{s}$ and about $t=465 \mathrm{~s}$ in figure 5 (middle). When $\langle h\rangle$ and $\left\langle u_{r m s}\right\rangle$ assume relatively large values, $h$ and $u_{\text {rms }}$ show strong fast fluctuations caused by the rotation of the humps, in addition to the $10 \mathrm{~s}$ scale temporal variations (figure $5 a$ ). However, the amplitudes of the fast oscillation of $h$ and $u_{r m s}$ are small during the elongation of the free surface because the shape of the surface is close to axisymmetric without humps. Just before the surface reaches the highest position $(t \simeq 317 \mathrm{~s})$, the humps are most clearly distinguished (Tasaka et al. 2008b). In this period, both the oscillation amplitudes of $h$ and $u_{r m s}$ are larger as also reflected in the slow-scale variations.

The RO (figure $5 b$ ) is a regular vertical oscillation of the free surface with period $\sim 10 \mathrm{~s}\left(\sim 100 \Omega^{-1}\right)$, which appears as a peak in the power spectrums of $u^{\prime}(r, t)$ (Tasaka et al. 2008a) and $h(t)$ (Suzuki et al. 2006). A typical RO can be observed at $320 \mathrm{~s}<t<380 \mathrm{~s}(355-375 \mathrm{~s}$ of this section is shown in magnification). The oscillation period of RO is much longer than the period of the rotation of the humps (about $0.35 \mathrm{~s}$ in this condition), corresponding to the main frequency in the power spectrums. The time series of the turbulent intensity $\left\langle u_{r m s}\right\rangle /(2 \pi \Omega R)$ in $\mathrm{RO}$ is 0.02 on average but it oscillates around the average (amplitude about 0.005 ; figure $5 b$ ). When $\left\langle u_{r m s}\right\rangle$ is large, the fluctuation amplitude of $u_{r m s}$ is also relatively large. The RO is similar to the state regularly observed at high speeds of rotation (the As region in figure $4 b$ ).

In FR the free surface rotates with a flatter shape without humps, however, the horizontal cross-section has $n=2$ symmetry. It is observed at $390 \mathrm{~s}<t<410 \mathrm{~s}$. The $\left\langle u_{r m s}\right\rangle /(2 \pi \Omega R)$ time series is roughly the same average as that in $\mathrm{RO}$, but it oscillates only little (figure $5 c$ ), because the humps are indistinct, the amplitude of the oscillation $h$ is small. However, the amplitude of the oscillation of $u_{r m s}$ is not small as the surface has a non-axisymmetric shape, causing strong mixing. Thus it may be concluded that the fluctuations in the flow field are mainly caused by the rotation of the free surface with the $n=2$ symmetry, while FR is similar to the state regularly observed in the $A s^{\prime}$ region in figure $4(b)$.

We have found that the typical sequences observed in $S w$ (RO, LTS and FR in figure 5 (bottom)) are similar to the characteristic states in the neighbourhood of the Reynolds numbers where $S w$ occurs: $A s, A s^{\prime}$ and $S y^{\prime}$ (figure $4 b$ ). Also, the Reynolds numbers for $S w$ close to the critical points for the laminar-to-turbulent $\left(R e=R e_{c}\right)$ and the turbulent-to-laminar $\left(R e=R e_{b}^{\prime}\right)$ transitions. The mechanism to sustain the $A s, A s^{\prime}$ and $S y^{\prime}$ states easily fluctuates due to the large turbulent intensity. Then, when the whole system is divided into the intrinsic laminar-turbulent transition dynamics and the noise (flow fluctuation), the effect of the noise will appear as a temporal shift in the critical points. In other words, the origin of the dynamic processes in the $S w$ region (switching among LTS, RO and FR) may be understood as an entrainment of quasi-steady states similar to $A s, A s^{\prime}$ and $S y^{\prime}$, and arising due to the noise.

\section{Conclusions}

Details of the occurrence of surface switching has been clarified both as a dynamic process and the bifurcation structure. Simultaneous measurements of the surface height and the velocity field showed that the process of the surface switching consists 
of two kinds of non-axisymmetric surface shapes (RO and FR states) as well as a dynamic transition between laminar and turbulent states (LTS state). The bifurcation diagram constructed by the turbulent intensity as a function of the Reynolds number suggests that the two flow states in the switching process, laminar and turbulent, are connected to two different branches of the turbulent intensity. The bifurcation diagram shows hysteresis; the two branches overlap at a region of Reynolds numbers. The quasi-steady states in the switching phenomenon may be traced back to the states on the bifurcation diagram in the neighbourhood of the switching region. It is noteworthy that the Reynolds numbers for the surface switching is close to the critical Reynolds numbers for the laminar-to-turbulent and turbulent-to-laminar transitions. A significant part of the mechanism of the surface switching can be understood as temporal shifts in the critical Reynolds numbers, arising from large flow fluctuations.

Repeated transitions between laminar and turbulent flows appear as a turbulent spot in the transition of the boundary layer (Perry, Lim \& Teh 1981), and as a puff or a slug in pipe flow (Wygnanski \& Champagne 1973). These transitions occur in spatially localized regions where the flow is turbulent. In the turbulent state in LTS, the flow is strongly disturbed in the whole region bounded by the free surface, and the shape is strongly correlated to the flow characteristics. Thus, the laminar-turbulent transition dynamics is coupled with the dynamics of the free surface. Although the turbulent spot, the puff and the slug do not interact with a free surface, the systems here may have part of the intrinsic laminar-turbulent transition dynamics in common. If this is so, an understanding of this system could help to solve problems in laminarturbulent transitions including the finite-lifetime problems in turbulence studies (Hof et al. 2006).

Surface tension plays an important role in the detachment of the surface, as suggested by the fact that the disk material changes the Reynolds number region of the surface switching. The surface detachment process has been visualized (Tasaka et al. $2008 b$ ), but a detailed mechanism of the surface instability, occurring at $R e=R e_{b}$, and that at the detachment of the surface, occurring at $R e=R e_{c}$, remain to be fully described. Recent experiments have shown that using a larger disk (smaller $\Delta R$ ) makes $R e_{c}$ smaller, and makes LTS occur more frequently, but without essential differences when compared with the phenomenon explained here. Presently, investigation is also made of the effect of the surface tension using silicone oil, which has a smaller surface tension, and also with liquid gallium, which has a larger surface tension: Results here suggest that a smaller Weber number enhances the breaking of the axisymmetry and there is an absence of surface switching (Tasaka, Yano \& Iima 2008c). The aspect ratio, $\mathscr{A}$, also affects the phenomenon: the temporally irregular LTS was observed at $0.47 \leqslant \mathscr{A} \leqslant 1.05$, and hysteresis appears at $\mathscr{A}<1$. At larger aspect ratios, the variation of the surface height in the process appears restricted by a larger pressure gradient, and thus the relative amplitude of the surface height variation becomes relatively smaller than that of the smaller $\mathscr{A}$. To understand the interfacial instability of the free surface attaching itself to the bottom, it will be necessary to investigate the flow in the boundary layer in detail. Comprehensive and detailed studies in this, including the investigations mentioned above, are in progress and will be reported separately.

This work is supported by Grant-Aided Research for Science, No. 18760116. The authors express thanks for this support. The authors also thank Professor Susumu Goto and Dr Gregory P. King for useful comments. 


\section{REFERENCES}

CoRwin, E. I. 2008 Granular flow in a rapidly rotated system with fixed walls. Phys. Rev. E 77, 031308.

Cross, A. \& Le Gal, P. 2002 Spatiotemporal intermittency in the torsional Couette flow between a rotating and a stationary disk. Phys. Fluids 14, 1038.

Gregory, N., Stuart, J. T. \& Walker, W. S. 1955 On the stability of three-dimensional boundary layers with application to the flow due to a rotating disk. Phil. Trans. R. Soc. Lond. Ser. A 248, 155-199.

Hof, B., Westerweel, J., Schneider, T. M. \& Eckhardt, B. 2006 Finite lifetime of turbulence in shear flows. Nature 443, 59-62.

Jansson, T. R. N., Haspang, M. P., Jensen, K. H., Hersen, P. \& Bohr, T. 2006 Polygons on a rotating fluids surface. Phys. Rev. Lett. 96, 174502.

von KÁrmán, T. 1921 Über laminare und turbulente Reibung. Z. Angew. Math. Mech. 1, 232-252.

Le Gal, P., Tasaka, Y., Nagao, J., Cros, A. \& Yamaguchi, K. 2007 A statistical study of spots in torsional Couette flow. J. Engng Math. 57, 289-302.

Lopez, M., Marques, F., Hirsa, A. H. \& Miraghaie, R. 2004 Symmetry breaking in free-surface cylinder flows. J. Fluid Mech. 502, 99-126.

Mashiko, T., Tsuji, Y., Mizuno, T. \& SANo, M. 2004 Instantaneous measurement of velocity fields in developed thermal turbulence in mercury. Phys. Rev. E 69, 036306.

Perry, A. E., Lim, T. T. \& TeH, E. W. 1981 A visual study of turbulent spot. J. Fluid Mech. 104, 387-405.

ReED, H. L. \& SARIC, W. S. 1989 Stability of three-dimensional boundary layers. Annu. Rev. Fluid Mech. 21, 235-284.

Shatrov, V., Gerbeth, G. \& Hermann, R. 2008 An alternating magnetic field driven flow in a spinning cylindrical container. J. Fluids Engng 130, 071201.

Suzuki, T., IIma, M. \& Hayase, Y. 2006 Surface switching of rotating fluid in a cylinder. Phys. Fluids 18, 101701.

TAKEDA, Y. 1995 Instantaneous velocity profile measurement by ultrasonic Doppler method. JSME Intl J. B38, 8-16.

TAKEDA, Y. 1996 Quasi-periodic state and transition to turbulence in a rotating Couette system. J. Fluid Mech. 389, 81-99.

Tasaka, Y., Iima, M. \& Ito, K. 2008 a Rotating flow transition related to surface switching. J. Phys.: Conf. Ser. 137, 012030.

TasaKa, Y., Ito, K. \& Imмa, M. 2008b Visualization of a rotating flow under large-deformed free surface using anisotropic flakes. J. Vis. 11-2, 163-172.

Tasaka, Y., Kon, S., Schouveiler, L. \& Le Gal, P. 2006 Hysteretic mode exchange in the wake of two circular cylinders in tandem. Phys. Fluids 18, 084104.

TASAKa, Y., Yano, K. \& Iima, M. 2008c Ultrasonic investigation of flow transition in surface switching of rotating fluid. Proceedings of the Sixth Intl Symp. on Ultrasonic Doppler Method. PDF of the paper is available on the web; http://isud6.fsr.cvut.cz/

Vatistas, G. H. 1990 A note on liquid vortex sloshing and Kelvin's equilibria. J. Fluid Mech. 217, 241-248.

Vatistas, G. H., Abderrahmane, H. A. \& Siddiqui, M. H. K. 2008 Experimental confirmation of Kelvin's equilibria. Phys. Rev. Lett. 100, 174503.

Wygnanski, I. J. \& Champagne, F. H. 1973 On transition in a pipe. Part 1. The origin of puffs and slugs and the flow in a turbulent slug. J. Fluid Mech. 59, 281-335. 